\title{
ANAESTHESIA FOR AUTOKERATOPLASTY $* \dagger$ SOME IMPORTANT CONSIDERATIONS ILLUSTRATED BY A CASE REPORT
}

\author{
BY \\ NORMAN SANDERS \\ Bascom Palmer Eye Institute, Miami, Florida
}

WHILE the use of autokeratoplasty has long been advocated as an excellent procedure, it is unusual for one to find a patient with the special circumstances allowing this operation. The candidate must, of course, have one blind eye with a healthy cornea, while the second eye has a diseased cornea with reasonable retinal function. The blind eye usually receives a homotransplant and when the first keratoplasty is completed, the patient's clear corneal button is used for autocorneal transplant in the second eye.

The clinical and theoretical reasons for the success of an autotransplant have been worked out and proven many times and will not be examined here.

The principal purpose of this paper is to urge general anaesthesia for this procedure. If this is not possible, an anaesthetist should be present throughout the procedure. The following case report will illustrate this point.

A 59-year-old coloured man came to the Bascom Palmer Eye Institute with a blind right eye and a clear cornea. Approximately 12 years previously, he had undergone two unsuccessful retinal detachment procedures which fortunately left the cornea entirely intact (Fig. 1).

FIG. 1.-Right eye, no perception of light, cornea normal.

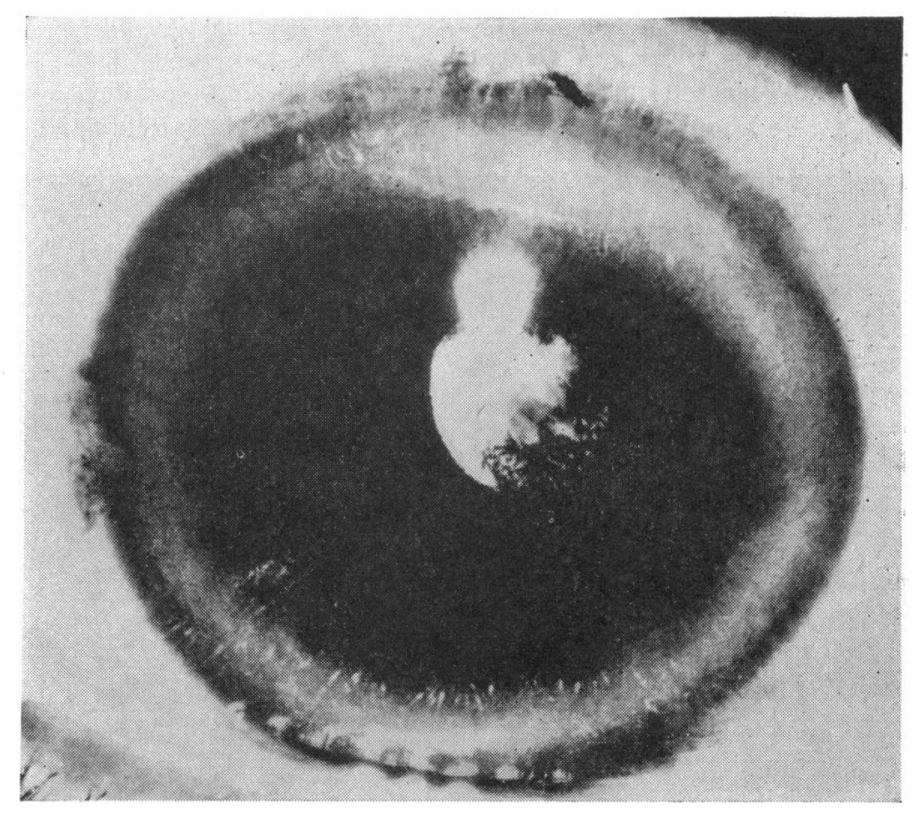

* Received for publication February 17, 1967

$\dagger$ Address for reprints: as above. 
In 1945, the left eye was struck with a nail and the cornea was lacerated, leaving an adherent leucoma. In 1958, he had an irido-capsulotomy, which left the cornea further scarred, so that when he was seen in May, 1966, the cornea was opaque and oedematous (Fig. 2).

Fig. 2.-Left eye, old corneal laceration followed by secondary intra-ocular surgery. Good perception of light and light projection.



On June 1, 1966, he was admitted for surgery but, because of complicating asthma, the anaesthetist refused to administer general anaesthesia. We did insist, however, that he should remain with the patient throughout the operation.

After we had removed the clear button from the blind eye, the patient went into an attack of status asthmaticus and his blood pressure dropped precipitously. The anaesthetist administered aminophylline, intravenous fluids, and Wyamine, and we proceeded with the operation. A donor homograft was then put into the blind right eye, using a running $8-0$ virgin silk suture. The left eye was given the autograft, using a running suture.

Several hours later the attack of asthma subsided and the patient's pulmonary status has since remained stable. Both transplants were clear, but after 6 weeks the right eye clouded (Fig. 3 ).

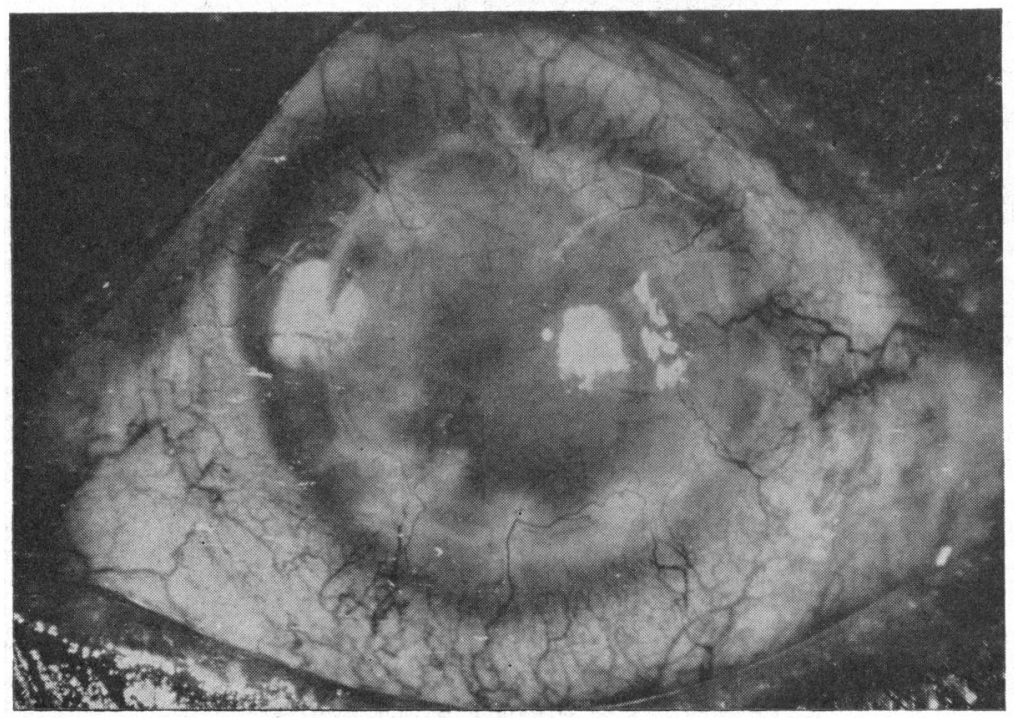

FIG. 3.-Right eye, cloudy homograft. 
The left autotransplant, however, has remained clear (Fig. 4) and 4 months after surgery the visual acuity is $20 / 200$, because of peripapillary retinal atrophy.

FIG. 4. - Left eye, clear autokeratoplasty, visual acuity $20 / 200$.

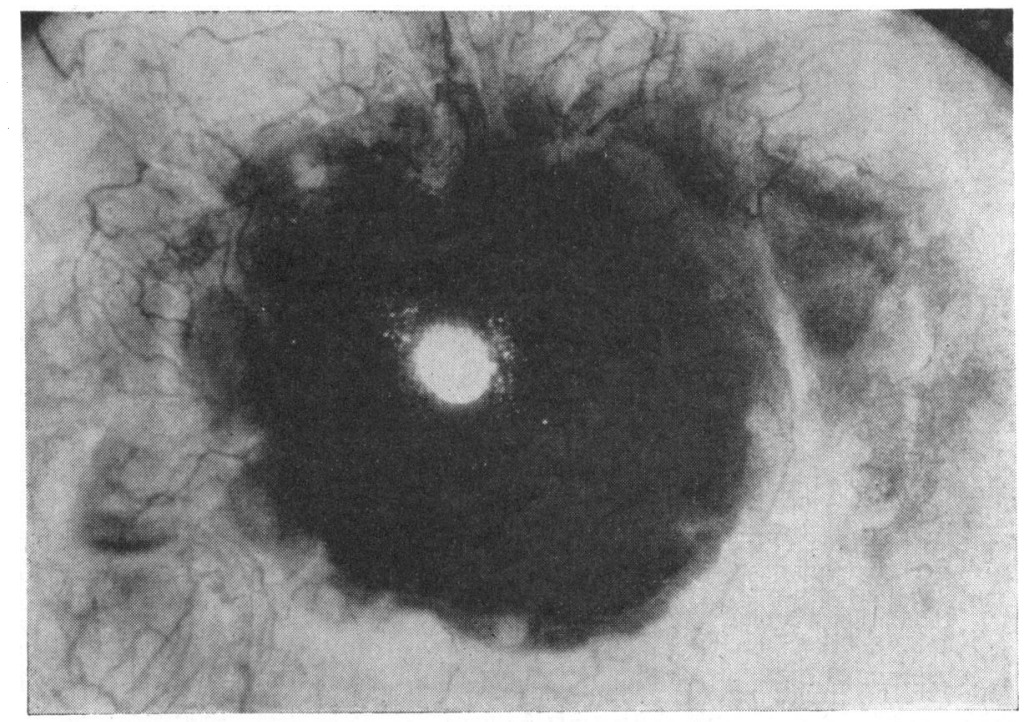

\section{Comment}

When it is possible, a corneal autotransplant is always preferable to homotransplantation. Once the operation is started, however, the procedure requires the patient to undergo two corneal transplants. (This is not applicable when an enucleation of the first eye is preferred, in which case a short time-lag is feasible.) Since the patient must lie quietly during the two operations, general anaesthesia should be administered if at all possible, to help ensure the completion of the operation. If for some reason, as in our case, local anaesthesia is necessary, it is imperative for an anaesthetist to be present to keep the patient quiet if he becomes agitated or restless. The importance of this cannot be over-emphasized. In fact, one should even consider placing a metal shield over the first eye as soon as the graft is removed and closing it after the autotransplant procedure is completed.

Apart from using extreme care in removing the host cornea, no time need be wasted with the first eye. It is obvious that there is no second or "back-up" autograft material, and it is imperative that this tissue be excised as perfectly as possible. Removal of the autograft button should be effected mainly with the trephine, since a cleaner excision can be done with this instrument than with scissors. Since the eye is blind, one need not worry about damaging the underlying structures. The removal must be very carefully completed with scissors, since it is difficult to remove the graft with the trephine only. The homograft is now placed into the defect. Several interrupted sutures and then a running stitch, can give an excellent water-tight wound. With practice, this can be an effective and rapid technique.

The second eye is now ready for surgery and the corneal transplant is then performed with the technique and care which would be given to any other transplant. 